\title{
Some Ramanujan-type circular summation formulas
}

Ji-Ke Ge ${ }^{1}$ and Qiu-Ming Luo ${ }^{2^{*}}$ (D)

Dedicated to Professor Hari Mohan Srivastava on his 80th birthday

*Correspondence:

luomath2007@163.com

${ }^{2}$ Department of Mathematics,

Chongqing Normal University, Chongqing Higher Education Mega

Center, Huxi Campus, Chongqing

401331, People's Republic of China

Full list of author information is

available at the end of the article

\begin{abstract}
In this paper, we give two Ramanujan-type circular summation formulas by applying the way of elliptic functions and the properties of theta functions. As applications, we obtain the corresponding imaginary transformation formulas for Ramanujan-type circular summations and some theta function identities.
\end{abstract}

MSC: Primary 11F27; secondary 11F20; 33E05

Keywords: Elliptic functions; Ramanujan-type circular summation; Theta functions; Theta function identities

\section{Introduction, preparation, and motivation}

The classical Jacobi four theta functions $\vartheta_{i}(z \mid \tau), i=1,2,3$, 4, with the notation of Tannery and Molk, are defined as follows.

Definition 1.1 (see, e.g., $[3,12,25])$ For $q=e^{\pi i \tau}, \operatorname{Im}(\tau)>0, z \in \mathbb{C}$.

$$
\begin{aligned}
& \vartheta_{1}(z \mid \tau)=-i q^{\frac{1}{4}} \sum_{n=-\infty}^{\infty}(-1)^{n} q^{n(n+1)} e^{(2 n+1) i z}, \\
& \vartheta_{2}(z \mid \tau)=q^{\frac{1}{4}} \sum_{n=-\infty}^{\infty} q^{n(n+1)} e^{(2 n+1) i z}, \\
& \vartheta_{3}(z \mid \tau)=\sum_{n=-\infty}^{\infty} q^{n^{2}} e^{2 n i z}, \\
& \vartheta_{4}(z \mid \tau)=\sum_{n=-\infty}^{\infty}(-1)^{n} q^{n^{2}} e^{2 n i z} .
\end{aligned}
$$

From the Jacobi theta functions (1.1)-(1.4), via the direct calculation, we have the following properties, respectively.

(c) The Author(s) 2020. This article is licensed under a Creative Commons Attribution 4.0 International License, which permits use sharing, adaptation, distribution and reproduction in any medium or format, as long as you give appropriate credit to the original author(s) and the source, provide a link to the Creative Commons licence, and indicate if changes were made. The images or other third party material in this article are included in the article's Creative Commons licence, unless indicated otherwise in a credit line the material. If material is not included in the article's Creative Commons licence and your intended use is not permitted by statutory regulation or exceeds the permitted use, you will need to obtain permission directly from the copyright holder. To view a copy of this licence, visit http://creativecommons.org/licenses/by/4.0/. 


\section{Proposition 1.2}

$$
\begin{array}{ll}
\vartheta_{1}(z+\pi \mid \tau)=-\vartheta_{1}(z \mid \tau), & \vartheta_{1}(z+\pi \tau \mid \tau)=-q^{-1} e^{-2 i z} \vartheta_{1}(z \mid \tau), \\
\vartheta_{2}(z+\pi \mid \tau)=-\vartheta_{2}(z \mid \tau), & \vartheta_{2}(z+\pi \tau \mid \tau)=q^{-1} e^{-2 i z} \vartheta_{2}(z \mid \tau), \\
\vartheta_{3}(z+\pi \mid \tau)=\vartheta_{3}(z \mid \tau), & \vartheta_{3}(z+\pi \tau \mid \tau)=q^{-1} e^{-2 i z} \vartheta_{3}(z \mid \tau), \\
\vartheta_{4}(z+\pi \mid \tau)=\vartheta_{4}(z \mid \tau), & \vartheta_{4}(z+\pi \tau \mid \tau)=-q^{-1} e^{-2 i z} \vartheta_{4}(z \mid \tau) .
\end{array}
$$

\section{Proposition 1.3}

$$
\begin{array}{ll}
\vartheta_{1}\left(z+\frac{\pi}{2} \mid \tau\right)=\vartheta_{2}(z \mid \tau), & \vartheta_{1}\left(z+\frac{\pi \tau}{2} \mid \tau\right)=i q^{-\frac{1}{4}} e^{-i z} \vartheta_{4}(z \mid \tau), \\
\vartheta_{2}\left(z+\frac{\pi}{2} \mid \tau\right)=-\vartheta_{1}(z \mid \tau), & \vartheta_{2}\left(z+\frac{\pi \tau}{2} \mid \tau\right)=q^{-\frac{1}{4}} e^{-i z} \vartheta_{3}(z \mid \tau), \\
\vartheta_{3}\left(z+\frac{\pi}{2} \mid \tau\right)=\vartheta_{4}(z \mid \tau), & \vartheta_{3}\left(z+\frac{\pi \tau}{2} \mid \tau\right)=q^{-\frac{1}{4}} e^{-i z} \vartheta_{2}(z \mid \tau), \\
\vartheta_{4}\left(z+\frac{\pi}{2} \mid \tau\right)=\vartheta_{3}(z \mid \tau), & \vartheta_{4}\left(z+\frac{\pi \tau}{2} \mid \tau\right)=i q^{-\frac{1}{4}} e^{-i z} \vartheta_{1}(z \mid \tau) .
\end{array}
$$

From (1.5)-(1.8), by applying induction, we easily obtain the following.

Lemma 1.4 For $n$ is a nonnegative integer, we have

$$
\begin{array}{ll}
\vartheta_{1}(z+n \pi \mid \tau)=(-1)^{n} \vartheta_{1}(z \mid \tau), & \vartheta_{1}(z+n \pi \tau \mid \tau)=(-1)^{n} q^{-n^{2}} e^{-2 n i z} \vartheta_{1}(z \mid \tau), \\
\vartheta_{2}(z+n \pi \mid \tau)=(-1)^{n} \vartheta_{2}(z \mid \tau), & \vartheta_{2}(z+n \pi \tau \mid \tau)=q^{-n^{2}} e^{-2 n i z} \vartheta_{2}(z \mid \tau), \\
\vartheta_{3}(z+n \pi \mid \tau)=\vartheta_{3}(z \mid \tau), & \vartheta_{3}(z+n \pi \tau \mid \tau)=q^{-n^{2}} e^{-2 n i z} \vartheta_{3}(z \mid \tau), \\
\vartheta_{4}(z+n \pi \mid \tau)=\vartheta_{4}(z \mid \tau), & \vartheta_{4}(z+n \pi \tau \mid \tau)=(-1)^{n} q^{-n^{2}} e^{-2 n i z} \vartheta_{4}(z \mid \tau) .
\end{array}
$$

From (1.9)-(1.16), we have the following lemmas.

Lemma 1.5 For $n$ is any positive integer, we have

$$
\begin{aligned}
& \vartheta_{1}\left(z+\frac{n \pi}{2} \mid \tau\right)= \begin{cases}i^{n} \vartheta_{1}(z \mid \tau), & \text { n is even, } \\
i^{n-1} \vartheta_{2}(z \mid \tau), & n \text { is odd, }\end{cases} \\
& \vartheta_{2}\left(z+\frac{n \pi}{2} \mid \tau\right)= \begin{cases}i^{n} \vartheta_{2}(z \mid \tau), & \text { n is even, } \\
-i^{n-1} \vartheta_{1}(z \mid \tau), & \text { n is odd, }\end{cases} \\
& \vartheta_{3}\left(z+\frac{n \pi}{2} \mid \tau\right)= \begin{cases}\vartheta_{3}(z \mid \tau), & n \text { is even, } \\
\vartheta_{4}(z \mid \tau), & n \text { is odd, }\end{cases} \\
& \vartheta_{4}\left(z+\frac{n \pi}{2} \mid \tau\right)= \begin{cases}\vartheta_{4}(z \mid \tau), & n \text { is even, } \\
\vartheta_{3}(z \mid \tau), & n \text { is odd. }\end{cases}
\end{aligned}
$$


Lemma 1.6 For $n$ is any positive integer, we have

$$
\begin{aligned}
& \vartheta_{1}\left(z+\frac{n \pi \tau}{2} \mid \tau\right)= \begin{cases}i^{n} q^{-\frac{n^{2}}{4}} e^{-n i z} \vartheta_{1}(z \mid \tau), & n \text { is even, } \\
i^{n} q^{-\frac{n^{2}}{4}} e^{-n i z} \vartheta_{4}(z \mid \tau), & n \text { is odd, }\end{cases} \\
& \vartheta_{2}\left(z+\frac{n \pi \tau}{2} \mid \tau\right)= \begin{cases}q^{-\frac{n^{2}}{4}} e^{-n i z} \vartheta_{2}(z \mid \tau), & n \text { is even, } \\
q^{-\frac{n^{2}}{4}} e^{-n i z} \vartheta_{3}(z \mid \tau), & n \text { is odd, }\end{cases} \\
& \vartheta_{3}\left(z+\frac{n \pi \tau}{2} \mid \tau\right)= \begin{cases}q^{-\frac{n^{2}}{4}} e^{-n i z} \vartheta_{3}(z \mid \tau), & n \text { is even, } \\
q^{-\frac{n^{2}}{4}} e^{-n i z} \vartheta_{2}(z \mid \tau), & n \text { is odd, }\end{cases} \\
& \vartheta_{4}\left(z+\frac{n \pi \tau}{2} \mid \tau\right)= \begin{cases}i^{n} q^{-\frac{n^{2}}{4}} e^{-n i z} \vartheta_{4}(z \mid \tau), & n \text { is even, } \\
i^{n} q^{-\frac{n^{2}}{4}} e^{-n i z} \vartheta_{1}(z \mid \tau), & n \text { is odd. }\end{cases}
\end{aligned}
$$

On page 54 in Ramanujan's lost notebook (see [21, p. 54, Entry 9.1.1], [2, p. 337]), Ramanujan recorded the following claim (without proof), which is now well known as Ramanujan's circular summation. The appellation circular summation was initiated by Son (see [2, p. 338]).

Theorem 1.7 (Ramanujan's circular summation) For each positive integer $n$ and $|a b|<1$,

$$
\sum_{-n / 2<r \leq n / 2}\left(\sum_{\substack{k=-\infty \\ k=r(\bmod n)}}^{\infty} a^{k(k+1) /(2 n)} b^{k(k-1) /(2 n)}\right)^{n}=f(a, b) F_{n}(a b),
$$

where

$$
F_{n}(q):=1+2 n q^{(n-1) / 2}+\cdots, \quad n \geq 3
$$

Ramanujan's theta function $f(a, b)$ is defined by

$$
f(a, b)=\sum_{n=-\infty}^{\infty} a^{n(n+1) / 2} b^{n(n-1) / 2}, \quad|a b|<1 .
$$

Chan, Liu, and $\mathrm{Ng}$ [10] proved that Theorem 1.7 is equivalent to the following form.

Theorem 1.8 (Ramanujan's circular summation) For each positive integer $n$,

$$
\sum_{k=0}^{n-1} q^{k^{2}} e^{2 k i z} \vartheta_{3}^{n}(z+k \pi \tau \mid n \tau)=\vartheta_{3}(z \mid \tau) F_{n}(\tau),
$$

where for $n \geq 3$,

$$
F_{n}(\tau)=1+2 n q^{n-1}+\cdots .
$$

Chan, Liu, and $\mathrm{Ng}[10]$ also showed that Theorem 1.8 is an equivalent of the theorem below by applying the Jacobi imaginary transformation formulas [25, p. 475]. They also proved that Theorem 1.9 is equivalent to Theorem 1.7. 
Theorem 1.9 (Ramanujan's circular summation) For any positive integer $n$, there exists a quantity $G_{n}(\tau)$ such that

$$
\sum_{k=0}^{n-1} \vartheta_{3}^{n}\left(z+\frac{k \pi}{n} \mid \tau\right)=G_{n}(\tau) \vartheta_{3}(n z \mid n \tau)
$$

where

$$
G_{n}(\tau)=\sqrt{n}(-i \tau)^{(1-n) / 2} F_{n}\left(-\frac{1}{n \tau}\right)
$$

Ramanujan's circular summation is an interesting subject in his notebook. On the subject of Ramanujan's circular summation and related theta function identities and their various extensions, a remarkably large number of investigations have appeared in the literature (see, for example, Andrews, Berndt, Rangachari, Ono, Ahlgren, Chua, Murayama, Son, Chan, Liu, Ng, Chan, Shen, Cai, Zhu, and Xu et al. [1, 2, 4-11, 13, 14, 16, 18-20, $22-$ $24,26,27,29,30])$.

Recently, Liu and Luo [15] obtained the alternating circular summation formulas of theta function $\vartheta_{3}(z \mid \tau)$. Luo [17] further generalized the results of Chan and Liu on Ramanujan's circular summation formula for theta functions $\vartheta_{3}(z \mid \tau)$ and deduced some alternating summation formulas of theta functions $\vartheta_{1}(z \mid \tau)$ and $\vartheta_{2}(z \mid \tau)$. Zhou and Luo [28] studied a variation for Ramanujan's circular summation of theta function $\vartheta_{4}(z \mid \tau)$, which here we call the Ramanujan-type circular summation.

Motivated by $[10,11]$, and $[15,17,28]$, by applying the theory of elliptic functions, we further investigate other two Ramanujan-type circular summations for theta functions $\vartheta_{1}(z \mid \tau)$ and $\vartheta_{2}(z \mid \tau)$, which are two variations of Ramanujan's circular summations (noting that is not alternating).

The paper is organized as follows: In the first section we display the definitions and properties of four theta functions. In the second section we show and prove two Ramanujantype circular summation formulas for theta functions $\vartheta_{1}(z \mid \tau)$ and $\vartheta_{2}(z \mid \tau)$ based on the theory and method of elliptic functions and properties of theta functions. In the third section we derive the corresponding imaginary transformation formulas of circular summation formulas by using the imaginary transformation formulas of $\vartheta_{1}(z \mid \tau)$ and $\vartheta_{2}(z \mid \tau)$. In the fourth section we give some further results and remarks.

\section{Two Ramanujan-type circular summation formulas}

In the present section, by applying the method for elliptic functions, we obtain two Ramanujan-type circular summation formulas of theta functions $\vartheta_{1}(z \mid \tau)$ and $\vartheta_{2}(z \mid \tau)$, respectively, which are two variations for Ramanujan's circular summation formulas. We now state our main results as follows.

Theorem 2.1 Let $n$ be even, $m$ be any positive integer, and $p$ be any integer. Also let $y_{1}, y_{2}, \ldots, y_{n}$ be any complex numbers. Then:

- When $y_{1}+y_{2}+\cdots+y_{n}=\frac{p \pi}{m}$, we have

$$
\sum_{k=0}^{m n-1} \prod_{j=1}^{n} \vartheta_{1}\left(z+y_{j}+\frac{k \pi}{m n} \mid \tau\right)=R_{1,1}^{(1)}\left(y_{1}, y_{2}, \ldots, y_{n} ; m, n, p ; \tau\right) \vartheta_{3}\left(m n z \mid m^{2} n \tau\right)
$$


where

$$
R_{1,1}^{(1)}\left(y_{1}, y_{2}, \ldots, y_{n} ; m, n, p ; \tau\right)=m n q^{-\frac{n}{4}} e^{\frac{p \pi i}{m}} \sum_{\substack{r_{1}, \ldots, r_{n}=-\infty \\ r_{1}+\cdots+r_{n}=\frac{n}{2}}}^{\infty} q^{r^{2}+\cdots+r_{n}^{2}} e^{-2 i\left(r_{1} y_{1}+\cdots+r_{n} y_{n}\right)}
$$

- When $y_{1}+y_{2}+\cdots+y_{n}=\frac{(2 p+1) \pi}{2 m}$, we have

$$
\sum_{k=0}^{m n-1} \prod_{j=1}^{n} \vartheta_{1}\left(z+y_{j}+\frac{k \pi}{m n} \mid \tau\right)=R_{1,1}^{(2)}\left(y_{1}, y_{2}, \ldots, y_{n} ; m, n, p ; \tau\right) \vartheta_{4}\left(m n z \mid m^{2} n \tau\right)
$$

where

$$
\begin{aligned}
& R_{1,1}^{(2)}\left(y_{1}, y_{2}, \ldots, y_{n} ; m, n, p ; \tau\right) \\
& =m n q^{-\frac{n}{4}} e^{\frac{(2 p+1) \pi i}{2 m}} \sum_{\substack{r_{1}, \ldots, r_{n}=-\infty \\
r_{1}+\cdots+r_{n}=\frac{n}{2}}}^{\infty} q^{r_{1}^{2}+\cdots+r_{n}^{2}} e^{-2 i\left(r_{1} y_{1}+\cdots+r_{n} y_{n}\right)} .
\end{aligned}
$$

Proof Let $f(z)$ be the left-hand side of (2.1) with $z \longmapsto \frac{z}{m n}, \tau \longmapsto \frac{\tau}{m^{2} n}$. We have

$$
f(z)=\sum_{k=0}^{m n-1} \prod_{j=1}^{n} \vartheta_{1}\left(\frac{z}{m n}+y_{j}+\frac{k \pi}{m n} \mid \frac{\tau}{m^{2} n}\right) .
$$

By (1.5), we easily obtain

$$
\begin{aligned}
f(z+\pi) & =\sum_{k=0}^{m n-1} \prod_{j=1}^{n} \vartheta_{1}\left(\frac{z+\pi}{m n}+y_{j}+\frac{k \pi}{m n} \mid \frac{\tau}{m^{2} n}\right) \\
& =\sum_{k=1}^{m n-1} \prod_{j=1}^{n} \vartheta_{1}\left(\frac{z}{m n}+y_{j}+\frac{k \pi}{m n} \mid \frac{\tau}{m^{2} n}\right)+(-1)^{n} \prod_{j=1}^{n} \vartheta_{1}\left(\frac{z}{m n}+y_{j} \mid \frac{\tau}{m^{2} n}\right) .
\end{aligned}
$$

Comparing (2.5) and (2.6), when $n$ is only even, we have

$$
f(z)=f(z+\pi)
$$

By (1.13) and noting that $n$ is even, we obtain

$$
\begin{aligned}
f(z+\pi \tau) & =\sum_{k=0}^{m n-1} \prod_{j=1}^{n} \vartheta_{1}\left(\frac{z+\pi \tau}{m n}+y_{j}+\frac{k \pi}{m n} \mid \frac{\tau}{m^{2} n}\right) \\
& =\sum_{k=0}^{m n-1} \prod_{j=1}^{n} \vartheta_{1}\left(\frac{z}{m n}+y_{j}+\frac{k \pi}{m n}+m \pi \frac{\tau}{m^{2} n} \mid \frac{\tau}{m^{2} n}\right) \\
& =\sum_{k=0}^{m n-1} \prod_{j=1}^{n}(-1)^{m} q^{-\frac{1}{n}} e^{-2 i m\left(\frac{z}{m n}+y_{j}+\frac{k \pi}{m n}\right)} \vartheta_{1}\left(\frac{z}{m n}+y_{j}+\frac{k \pi}{m n} \mid \frac{\tau}{m^{2} n}\right) \\
& =(-1)^{m n} q^{-1} e^{-2 i z} e^{-2 k \pi i} e^{-2 i m\left(y_{1}+y_{2}+\cdots+y_{n}\right)} f(z) \\
& =q^{-1} e^{-2 i z} e^{-2 i m\left(y_{1}+y_{2}+\cdots+y_{n}\right)} f(z) .
\end{aligned}
$$


- When $y_{1}+y_{2}+\cdots+y_{n}=\frac{p \pi}{m}$ in (2.8), we have

$$
f(z+\pi \tau)=q^{-1} e^{-2 i z} f(z)
$$

We construct the function $\frac{f(z)}{\vartheta_{3}(z \mid \tau)}$. By (1.7), (2.7), and (2.9), we find that the function $\frac{f(z)}{\vartheta_{3}(z \mid \tau)}$ is an elliptic function with double periods $\pi$ and $\pi \tau$, and has only a simple pole at $z=\frac{\pi}{2}+\frac{\pi \tau}{2}$ in the period parallelogram. Hence the function $\frac{f(z)}{\vartheta_{3}(z \mid \tau)}$ is a constant, say $C_{1,1}^{(1)}\left(y_{1}, y_{2}, \ldots, y_{n} ; \tau\right)$, we have

$$
f(z)=C_{1,1}^{(1)}\left(y_{1}, y_{2}, \ldots, y_{n} ; \tau\right) \vartheta_{3}(z \mid \tau)
$$

or, alternatively,

$$
\sum_{k=0}^{m n-1} \prod_{j=1}^{n} \vartheta_{1}\left(\frac{z}{m n}+y_{j}+\frac{k \pi}{m n} \mid \frac{\tau}{m^{2} n}\right)=C_{1,1}^{(1)}\left(y_{1}, y_{2}, \ldots, y_{n} ; \tau\right) \vartheta_{3}(z \mid \tau)
$$

Letting

$$
z \longmapsto m n z \quad \text { and } \quad \tau \longmapsto m^{2} n \tau
$$

in (2.10), and then setting

$$
R_{1,1}^{(1)}\left(y_{1}, y_{2}, \ldots, y_{n} ; m, n, p ; \tau\right)=C_{1,1}^{(1)}\left(y_{1}, y_{2}, \ldots, y_{n} ; m^{2} n \tau\right)
$$

we arrive at (2.1)

Setting

$$
z \longmapsto z+y_{j}+\frac{k \pi}{m n}
$$

in (1.1), by some simple calculation and noting that $n$ is even, we obtain

$$
\begin{aligned}
\prod_{j=1}^{n} \vartheta_{1}\left(z+y_{j}+\frac{k \pi}{m n} \mid \tau\right)= & i^{n} q^{\frac{n}{4}} e^{i\left(y_{1}+y_{2}+\cdots+y_{n}\right)} \sum_{r_{1}, \ldots, r_{n}=-\infty}^{\infty}(-1)^{r_{1}+\cdots+r_{n}} q^{r_{1}^{2}+\cdots+r_{n}^{2}+r_{1}+\cdots+r_{n}} \\
& \times e^{\left(2 r_{1}+\cdots+2 r_{n}+n\right) i z} e^{2 i\left(r_{1} y_{1}+\cdots+r_{n} y_{n}\right)} e^{\frac{k \pi i}{m n}\left(2 r_{1}+\cdots+2 r_{n}+n\right)} .
\end{aligned}
$$

Setting

$$
z \longmapsto m n z \quad \text { and } \quad \tau \longmapsto m^{2} n \tau
$$

in (1.3), we get

$$
\vartheta_{3}\left(m n z \mid m^{2} n \tau\right)=\sum_{r=-\infty}^{\infty} q^{m^{2} n r^{2}} e^{2 m n r i z}
$$


Substituting (2.11) and (2.12) into (2.1), we have

$$
\begin{aligned}
& R_{1,1}^{(1)}\left(y_{1}, y_{2}, \ldots, y_{n} ; m, n, p ; \tau\right) \sum_{r=-\infty}^{\infty} q^{m^{2} n r^{2}} e^{2 m n r i z} \\
& =i^{n} q^{\frac{n}{4}} e^{i\left(y_{1}+y_{2}+\cdots+y_{n}\right)} \sum_{k=0}^{m n-1} \sum_{r_{1}, \ldots, r_{n}=-\in f t y}^{\infty}(-1)^{r_{1}+\cdots+r_{n}} q^{r_{1}^{2}+\cdots+r_{n}^{2}+r_{1}+\cdots+r_{n}} \\
& \quad \times e^{\left(2 r_{1}+\cdots+2 r_{n}+n\right) i z} e^{2 i\left(r_{1} y_{1}+\cdots+r_{n} y_{n}\right)} e^{\frac{k \pi i}{m n}\left(2 r_{1}+\cdots+2 r_{n}+n\right)}
\end{aligned}
$$

Equating the constants of both sides of (2.13) and noting the condition $y_{1}+y_{2}+\cdots+y_{n}=$ $\frac{p \pi}{m}$, we get $(2.2)$.

- When $y_{1}+y_{2}+\cdots+y_{n}=\frac{(2 p+1) \pi}{2 m}$ in (2.8), we have

$$
f(z+\pi \tau)=-q^{-1} e^{-2 i z} f(z)
$$

We construct the function $\frac{f(z)}{\vartheta_{4}(z \mid \tau)}$. By (1.8), (2.7), and (2.14), we find that the function $\frac{f(z)}{\vartheta_{4}(z \mid \tau)}$ is an elliptic function with double periods $\pi$ and $\pi \tau$, and has only a simple pole at $z=\frac{\pi \tau}{2}$ in the period parallelogram. Hence the function $\frac{f(z)}{\vartheta_{4}(z \mid \tau)}$ is a constant, say $C_{1,1}^{(2)}\left(y_{1}, y_{2}, \ldots, y_{n} ; \tau\right)$, we have

$$
f(z)=C_{1,1}^{(2)}\left(y_{1}, y_{2}, \ldots, y_{n} ; \tau\right) \vartheta_{4}(z \mid \tau)
$$

or, equivalently,

$$
\sum_{k=0}^{m n-1} \prod_{j=1}^{n} \vartheta_{1}\left(\frac{z}{m n}+y_{j}+\frac{k \pi}{m n} \mid \frac{\tau}{m^{2} n}\right)=C_{11}^{(1)}\left(y_{1}, y_{2}, \ldots, y_{n} ; \tau\right) \vartheta_{4}(z \mid \tau)
$$

Letting

$$
z \longmapsto m n z \quad \text { and } \quad \tau \longmapsto m^{2} n \tau
$$

in (2.15), and then setting

$$
R_{1,1}^{(2)}\left(y_{1}, y_{2}, \ldots, y_{n} ; m, n, p ; \tau\right)=C_{11}^{(2)}\left(y_{1}, y_{2}, \ldots, y_{n} ; m^{2} n \tau\right)
$$

we arrive at (2.3).

A similar proof as that of (2.2). By using (1.1) and (1.4) in (2.3), and noting that $n$ is even and the condition $y_{1}+y_{2}+\cdots+y_{n}=\frac{(2 p+1) \pi}{2 m}$, we can obtain (2.4). This proof is complete.

Theorem 2.2 Let $n$ be even, $m$ be any positive integer, and $p$ be any integer. Also let $y_{1}, y_{2}, \ldots, y_{n}$ be any complex numbers. Then

- When $y_{1}+y_{2}+\cdots+y_{n}=\frac{p \pi}{m}$, we have

$$
\sum_{k=0}^{m n-1} \prod_{j=1}^{n} \vartheta_{2}\left(z+y_{j}+\frac{k \pi}{m n} \mid \tau\right)=R_{2,2}^{(1)}\left(y_{1}, y_{2}, \ldots, y_{n} ; m, n, p ; \tau\right) \vartheta_{3}\left(m n z \mid m^{2} n \tau\right)
$$


where

$$
R_{2,2}^{(1)}\left(y_{1}, y_{2}, \ldots, y_{n} ; m, n, p ; \tau\right)=m n q^{-\frac{n}{4}} e^{\frac{p \pi i}{m}} \sum_{\substack{r_{1}, \ldots, r_{n}=-\infty \\ r_{1}+\cdots+r_{n}=\frac{n}{2}}}^{\infty} q^{r^{2}+\cdots+r_{n}^{2}} e^{-2 i\left(r_{1} y_{1}+\cdots+r_{n} y_{n}\right)}
$$

- When $y_{1}+y_{2}+\cdots+y_{n}=\frac{(2 p+1) \pi}{2 m}$, we have

$$
\sum_{k=0}^{m n-1} \prod_{j=1}^{n} \vartheta_{2}\left(z+y_{j}+\frac{k \pi}{m n} \mid \tau\right)=R_{2,2}^{(2)}\left(y_{1}, y_{2}, \ldots, y_{n} ; m, n, p ; \tau\right) \vartheta_{4}\left(m n z \mid m^{2} n \tau\right)
$$

where

$$
\begin{aligned}
& R_{2,2}^{(2)}\left(y_{1}, y_{2}, \ldots, y_{n} ; m, n, p ; \tau\right) \\
& =m n q^{-\frac{n}{4}} e^{\frac{(2 p+1) \pi i}{2 m}} \sum_{\substack{r_{1}, \ldots, r_{n}=-\infty \\
r_{1}+\cdots+r_{n}=\frac{n}{2}}}^{\infty} q^{r_{1}^{2}+\cdots+r_{n}^{2}} e^{-2 i\left(r_{1} y_{1}+\cdots+r_{n} y_{n}\right)}
\end{aligned}
$$

Proof For $n$ is even, hence $\frac{m n}{2}$ is a positive integer.

Setting

$$
z \longmapsto z+\frac{\pi}{2}
$$

in equation (2.1) of Theorem 2.1 and applying properties (1.9) and (1.19), we arrive at formula (2.16) of Theorem 2.2.

Setting

$$
z \longmapsto z+\frac{\pi}{2}
$$

in equation (2.3) of Theorem 2.1 and applying properties (1.9) and (1.20), we arrive at formula (2.18) of Theorem 2.2.

Clearly, we consider that both $R_{1,1}^{(1)}\left(y_{1}, y_{2}, \ldots, y_{n} ; m, n, p ; \tau\right)$ and $R_{1,1}^{(2)}\left(y_{1}, y_{2}, \ldots, y_{n} ; m, n, p ; \tau\right)$ are independent of $z$, therefore we have

$$
R_{2,2}^{(1)}\left(y_{1}, y_{2}, \ldots, y_{n} ; m, n, p ; \tau\right)=R_{1,1}^{(1)}\left(y_{1}, y_{2}, \ldots, y_{n} ; m, n, p ; \tau\right)
$$

and

$$
R_{2,2}^{(2)}\left(y_{1}, y_{2}, \ldots, y_{n} ; m, n, p ; \tau\right)=R_{1,1}^{(2)}\left(y_{1}, y_{2}, \ldots, y_{n} ; m, n, p ; \tau\right)
$$

The proof is complete.

\section{The imaginary transformation formulas for Ramanujan-type circular summations}

In the present section, we first derive the corresponding imaginary transformation formulas of Theorem 2.1 by applying the imaginary transformations for theta functions. Some theta function identities are also shown. 
Theorem 3.1 Let $n$ be even, $m$ be any positive integer, and $p$ be any integer. Also let $y_{1}, y_{2}, \ldots, y_{n}$ be any complex numbers. Then

- When $y_{1}+y_{2}+\cdots+y_{n}=m n p$, we have

$$
\begin{aligned}
& \sum_{k=0}^{m n-1} q^{k^{2}+2 k p} e^{2(k+p) i z} \prod_{j=1}^{n} \vartheta_{1}\left(m z+y_{j} \pi \tau+m k \pi \tau \mid m^{2} n \tau\right) \\
& \quad=F_{1,1}^{(1)}\left(y_{1}, y_{2}, \ldots, y_{n} ; m, n, p ; \tau\right) \vartheta_{3}(z \mid \tau),
\end{aligned}
$$

where

$$
\begin{aligned}
& F_{1,1}^{(1)}\left(y_{1}, y_{2}, \ldots, y_{n} ; m, n, p ; \tau\right) \\
& =\frac{(\sqrt{-i})^{1-3 n} \sqrt{\tau^{1-n}}}{(m \sqrt{n})^{n}} q^{-\frac{y_{1}^{2}+\cdots+y_{n}^{2}}{m^{2} n}} R_{1,1}^{(1)}\left(\frac{y_{1} \pi}{m^{2} n}, \frac{y_{2} \pi}{m^{2} n}, \ldots, \frac{y_{n} \pi}{m^{2} n} ; m, n, p ;-\frac{1}{m^{2} n \tau}\right), \\
& F_{1,1}^{(1)}\left(y_{1}, y_{2}, \ldots, y_{n} ; m, n, p ; \tau\right) \\
& =i^{n} \sum_{k=0}^{m n-1} q^{-\left(k+\frac{m n}{2}\right)^{2}} \\
& \quad \times \sum_{2 m\left(r_{1}+\cdots+r_{n}\right)=m n+2(k+p)}^{\infty}(-1)^{r_{1}+\cdots+r_{n}} q^{m^{2} n\left(r_{1}^{2}+\cdots+r_{n}^{2}\right)-2\left(r_{1} y_{1}+\cdots+r_{n} y_{n}\right) .} .
\end{aligned}
$$

- When $y_{1}+y_{2}+\cdots+y_{n}=\frac{(2 p+1) m n}{2}$, we have

$$
\begin{aligned}
& \sum_{k=0}^{m n-1} q^{k^{2}+k(2 p+1)} e^{(2 k+2 p+1) i z} \prod_{j=1}^{n} \vartheta_{1}\left(m z+y_{j} \pi \tau+m k \pi \tau \mid m^{2} n \tau\right) \\
& \quad=F_{1,1}^{(2)}\left(y_{1}, y_{2}, \ldots, y_{n} ; m, n, p ; \tau\right) \vartheta_{2}(z \mid \tau),
\end{aligned}
$$

where

$$
\begin{aligned}
& F_{1,1}^{(2)}\left(y_{1}, y_{2}, \ldots, y_{n} ; m, n, p ; \tau\right) \\
& =\frac{(\sqrt{-i})^{1-3 n} \sqrt{\tau^{1-n}}}{(m \sqrt{n})^{n}} q^{-\frac{y_{1}^{2}+\cdots+y_{n}^{2}}{m^{2} n}} R_{1,1}^{(2)}\left(\frac{y_{1} \pi}{m^{2} n}, \frac{y_{2} \pi}{m^{2} n}, \ldots, \frac{y_{n} \pi}{m^{2} n} ; m, n, p ;-\frac{1}{m^{2} n \tau}\right), \\
& F_{1,1}^{(2)}\left(y_{1}, y_{2}, \ldots, y_{n} ; m, n, p ; \tau\right) \\
& =i^{n} \sum_{k=0}^{m n-1} q^{-\left(k+\frac{m n}{2}-\frac{1}{2}\right)^{2}} \\
& \quad \times \sum_{\substack{r_{1}, \ldots, r_{n}=-\infty \\
2 m\left(r_{1}+\cdots+r_{n}\right)=m n+2(k+p)}}^{\infty}(-1)^{r_{1}+\cdots+r_{n}} q^{m^{2} n\left(r_{1}^{2}+\cdots+r_{n}^{2}\right)-2\left(r_{1} y_{1}+\cdots+r_{n} y_{n}\right)} .
\end{aligned}
$$


Proof In (2.1) making the transformations $\tau \longmapsto-\frac{1}{m^{2} n \tau}$, and then $z \longmapsto \frac{z}{m n \tau}$ and $y_{j} \longmapsto \frac{y_{j} \pi}{m^{2} n}$ for $j=1,2, \ldots, n$, then equation (2.1) becomes

$$
\begin{aligned}
& \sum_{k=0}^{m n-1} \prod_{j=1}^{n} \vartheta_{1}\left(\frac{m z+y_{j} \pi \tau+m k \pi \tau}{m^{2} n \tau} \mid-\frac{1}{m^{2} n \tau}\right) \\
& \quad=R_{1,1}^{(1)}\left(\frac{y_{1} \pi}{m^{2} n}, \ldots, \frac{y_{n} \pi}{m^{2} n} ; m, n, p ;-\frac{1}{m^{2} n \tau}\right) \vartheta_{3}\left(\frac{z}{\tau} \mid-\frac{1}{\tau}\right) .
\end{aligned}
$$

Applying the imaginary transformations formulas (see, e.g., $[3,12,25])$

$$
\vartheta_{1}\left(\frac{z}{\tau} \mid-\frac{1}{\tau}\right)=-i \sqrt{-i \tau} e^{i \frac{z^{2}}{\pi \tau}} \vartheta_{1}(z \mid \tau) \quad \text { and } \quad \vartheta_{3}\left(\frac{z}{\tau} \mid-\frac{1}{\tau}\right)=\sqrt{-i \tau} e^{\frac{i z^{2}}{\pi \tau}} \vartheta_{3}(z \mid \tau)
$$

to the above equation (3.7), via the suitable substitutions of the variables $z$ and $\tau$ and noting that $y_{1}+y_{2}+\cdots+y_{n}=m n p$ and simplifying, we thus obtain (3.1) and (3.2). Applying the series expressions of $\vartheta_{1}(z \mid \tau)$ and $\vartheta_{3}(z \mid \tau)$ in (3.1), via direct calculation, we obtain (3.3).

In the same manner, using the imaginary transformations formulas

$$
\vartheta_{1}\left(\frac{z}{\tau} \mid-\frac{1}{\tau}\right)=-i \sqrt{-i \tau} e^{\frac{i z^{2}}{\pi \tau}} \vartheta_{1}(z \mid \tau) \quad \text { and } \quad \vartheta_{4}\left(\frac{z}{\tau} \mid-\frac{1}{\tau}\right)=\sqrt{-i \tau} e^{i \frac{z^{2}}{\pi \tau}} \vartheta_{2}(z \mid \tau)
$$

to (2.3) and noting that $y_{1}+y_{2}+\cdots+y_{n}=\frac{(2 p+1) m n}{2}$, we can prove formulas (3.4), (3.5), and (3.6), respectively. Therefore we complete the proof of Theorem 3.1.

Similarly, by applying the imaginary transformations formulas

$$
\vartheta_{2}\left(\frac{z}{\tau} \mid-\frac{1}{\tau}\right)=\sqrt{-i \tau} e^{\frac{i z^{2}}{\pi \tau}} \vartheta_{4}(z \mid \tau) \quad \text { and } \quad \vartheta_{3}\left(\frac{z}{\tau} \mid-\frac{1}{\tau}\right)=\sqrt{-i \tau} e^{\frac{i z^{2}}{\pi \tau}} \vartheta_{3}(z \mid \tau)
$$

we can obtain the following imaginary transformation formulas of Theorem 2.2.

Theorem 3.2 Let $n$ be even, $m$ be any positive integer, and $p$ be any integer. Also let $y_{1}, y_{2}, \ldots, y_{n}$ be any complex numbers. Then

- When $y_{1}+y_{2}+\cdots+y_{n}=$ mnp, we have

$$
\begin{aligned}
& \sum_{k=0}^{m n-1} q^{k^{2}+2 k p} e^{2(k+p) i z} \prod_{j=1}^{n} \vartheta_{4}\left(m z+y_{j} \pi \tau+m k \pi \tau \mid m^{2} n \tau\right) \\
& \quad=F_{2,2}^{(1)}\left(y_{1}, y_{2}, \ldots, y_{n} ; m, n, p ; \tau\right) \vartheta_{3}(z \mid \tau),
\end{aligned}
$$

where

$$
\begin{aligned}
& F_{2,2}^{(1)}\left(y_{1}, y_{2}, \ldots, y_{n} ; m, n, p ; \tau\right) \\
& \quad=\frac{(-i \tau)^{\frac{1-n}{2}}}{\left(m^{2} n\right)^{\frac{n}{2}}} q^{-\frac{y_{1}^{2}+\cdots+y_{n}^{2}}{m^{2} n}} R_{2,2}^{(1)}\left(\frac{y_{1} \pi}{m^{2} n}, \frac{y_{2} \pi}{m^{2} n}, \ldots, \frac{y_{n} \pi}{m^{2} n} ; m, n, p ;-\frac{1}{m^{2} n \tau}\right),
\end{aligned}
$$




$$
\begin{aligned}
& F_{2,2}^{(1)}\left(y_{1}, y_{2}, \ldots, y_{n} ; m, n, p ; \tau\right) \\
& \quad=\sum_{k=0}^{m n-1} \sum_{\substack{r_{1}, \ldots, r_{n}=-\infty \\
m\left(r_{1}+\cdots+r_{n}\right)=k+p}}^{\infty}(-1)^{r_{1}+\cdots+r_{n}} q^{m^{2} n\left(r_{1}^{2}+\cdots+r_{n}^{2}\right)-2\left(r_{1} y_{1}+\cdots+r_{n} y_{n}\right)-k^{2}}
\end{aligned}
$$

- When $y_{1}+y_{2}+\cdots+y_{n}=\frac{(2 p+1) m n}{2}$, we have

$$
\begin{aligned}
& \sum_{k=0}^{m n-1} q^{k^{2}+k(2 p+1)} e^{(2 k+2 p+1) i z} \prod_{j=1}^{n} \vartheta_{4}\left(m z+y_{j} \pi \tau+m k \pi \tau \mid m^{2} n \tau\right) \\
& \quad=F_{2,2}^{(2)}\left(y_{1}, y_{2}, \ldots, y_{n} ; m, n, p ; \tau\right) \vartheta_{2}(z \mid \tau)
\end{aligned}
$$

where

$$
\begin{aligned}
& F_{2,2}^{(2)}\left(y_{1}, y_{2}, \ldots, y_{n} ; m, n, p ; \tau\right) \\
& \quad=\frac{(-i \tau)^{\frac{1-n}{2}}}{\left(m^{2} n\right)^{\frac{n}{2}}} q^{-\frac{y_{1}^{2}+\cdots+y_{n}^{2}}{m^{2} n}} R_{2,2}^{(2)}\left(\frac{y_{1} \pi}{m^{2} n}, \frac{y_{2} \pi}{m^{2} n}, \ldots, \frac{y_{n} \pi}{m^{2} n} ; m, n, p ;-\frac{1}{m^{2} n \tau}\right) \\
& F_{2,2}^{(2)}\left(y_{1}, y_{2}, \ldots, y_{n} ; m, n, p ; \tau\right) \\
& \quad=\sum_{k=0}^{m n-1} \sum_{\substack{r_{1}, \ldots, r_{n}=-\infty \\
m\left(r_{1}+\cdots+r_{n}\right)=k+p}}^{\infty}(-1)^{r_{1}+\cdots+r_{n}} q^{m^{2} n\left(r_{1}^{2}+\cdots+r_{n}^{2}\right)-2\left(r_{1} y_{1}+\cdots+r_{n} y_{n}\right)-\left(k-\frac{1}{2}\right)^{2}}
\end{aligned}
$$

Taking $p=0$ in Theorem 3.1 .

Corollary 3.3 Let $n$ be even, $m$ be any positive integer. Also let $y_{1}, y_{2}, \ldots, y_{n}$ be any complex numbers. Then

- When $y_{1}+y_{2}+\cdots+y_{n}=0$, we have

$$
\begin{aligned}
& \sum_{k=0}^{m n-1} q^{k^{2}} e^{2 k i z} \prod_{j=1}^{n} \vartheta_{1}\left(m z+y_{j} \pi \tau+m k \pi \tau \mid m^{2} n \tau\right) \\
& =F_{1,1}^{(1)}\left(y_{1}, y_{2}, \ldots, y_{n} ; m, n ; \tau\right) \vartheta_{3}(z \mid \tau),
\end{aligned}
$$

where

$$
\begin{aligned}
& F_{1,1}^{(1)}\left(y_{1}, y_{2}, \ldots, y_{n} ; m, n ; \tau\right) \\
& \quad=\frac{(\sqrt{-i})^{1-3 n} \sqrt{\tau^{1-n}}}{(m \sqrt{n})^{n}} q^{-\frac{y_{1}^{2}+\cdots+y_{n}^{2}}{m^{2} n}} R_{1,1}^{(1)}\left(\frac{y_{1} \pi}{m^{2} n}, \frac{y_{2} \pi}{m^{2} n}, \ldots, \frac{y_{n} \pi}{m^{2} n} ; m, n ;-\frac{1}{m^{2} n \tau}\right), \\
& F_{1,1}^{(1)}\left(y_{1}, y_{2}, \ldots, y_{n} ; m, n ; \tau\right) \\
& =i^{n} \sum_{k=0}^{m n-1} q^{-\left(k+\frac{m n}{2}\right)^{2}} \sum_{\substack{r_{1}, \ldots, r_{n}=-\infty \\
2 m\left(r_{1}+\cdots+r_{n}\right)=m n+2 k}}^{\infty}(-1)^{r_{1}+\cdots+r_{n}} q^{m^{2} n\left(r_{1}^{2}+\cdots+r_{n}^{2}\right)-2\left(r_{1} y_{1}+\cdots+r_{n} y_{n}\right)} .
\end{aligned}
$$


- When $y_{1}+y_{2}+\cdots+y_{n}=\frac{m n}{2}$, we have

$$
\begin{aligned}
& \sum_{k=0}^{m n-1} q^{k^{2}+k} e^{(2 k+1) i z} \prod_{j=1}^{n} \vartheta_{1}\left(m z+y_{j} \pi \tau+m k \pi \tau \mid m^{2} n \tau\right) \\
& =F_{1,1}^{(2)}\left(y_{1}, y_{2}, \ldots, y_{n} ; m, n ; \tau\right) \vartheta_{2}(z \mid \tau),
\end{aligned}
$$

where

$$
\begin{aligned}
& F_{1,1}^{(2)}\left(y_{1}, y_{2}, \ldots, y_{n} ; m, n ; \tau\right) \\
& =\frac{(\sqrt{-i})^{1-3 n} \sqrt{\tau^{1-n}}}{(m \sqrt{n})^{n}} q^{-\frac{y_{1}^{2}+\cdots+y_{n}^{2}}{m^{2} n}} R_{1,1}^{(2)}\left(\frac{y_{1} \pi}{m^{2} n}, \frac{y_{2} \pi}{m^{2} n}, \ldots, \frac{y_{n} \pi}{m^{2} n} ; m, n ;-\frac{1}{m^{2} n \tau}\right), \\
& F_{1,1}^{(2)}\left(y_{1}, y_{2}, \ldots, y_{n} ; m, n ; \tau\right) \\
& =i^{n} \sum_{k=0}^{m n-1} q^{-\left(k+\frac{m n}{2}-\frac{1}{2}\right)^{2}} \\
& \quad \times \sum_{\substack{r_{1}, \ldots, r_{n}=-\infty \\
2 m\left(r_{1}+\cdots+r_{n}\right)=m n+2 k}}^{\infty}(-1)^{r_{1}+\cdots+r_{n}} q^{m^{2} n\left(r_{1}^{2}+\cdots+r_{n}^{2}\right)-2\left(r_{1} y_{1}+\cdots+r_{n} y_{n}\right)} .
\end{aligned}
$$

Corollary 3.4 Let $n$ be even and $m$ be any positive integer. Then

$$
\begin{aligned}
& \sum_{k=0}^{m n-1} q^{k^{2}} e^{2 k i z} \vartheta_{1}^{n}\left(m z+m k \pi \tau \mid m^{2} n \tau\right)=F_{1,1}^{(1)}(m, n ; \tau) \vartheta_{3}(z \mid \tau), \\
& \sum_{k=0}^{m n-1} q^{k^{2}+k} e^{(2 k+1) i z} \prod_{j=1}^{n} \vartheta_{1}\left(m z+\frac{m \pi \tau}{2}+m k \pi \tau \mid m^{2} n \tau\right)=F_{1,1}^{(2)}(m, n ; \tau) \vartheta_{2}(z \mid \tau),
\end{aligned}
$$

where

$$
\begin{aligned}
& F_{1,1}^{(1)}(m, n ; \tau)=\frac{(\sqrt{-i})^{1-3 n} \sqrt{\tau^{1-n}}}{(m \sqrt{n})^{n}} R_{1,1}^{(1)}\left(m, n ;-\frac{1}{m^{2} n \tau}\right) \\
& F_{1,1}^{(1)}(m, n ; \tau)=i^{n} \sum_{k=0}^{m n-1} q^{-\left(k+\frac{m n}{2}\right)^{2}} \sum_{\substack{r_{1}, \ldots, r_{n}=-\infty \\
2 m\left(r_{1}+\cdots+r_{n}\right)=m n+2 k}}^{\infty}(-1)^{r_{1}+\cdots+r_{n}} q^{m^{2} n\left(r_{1}^{2}+\cdots+r_{n}^{2}\right)}, \\
& F_{1,1}^{(2)}(m, n ; \tau)=\frac{(\sqrt{-i})^{1-3 n} \sqrt{\tau^{1-n}}}{(m \sqrt{n})^{n}} q^{-\frac{1}{4}} R_{1,1}^{(2)}\left(m, n ;-\frac{1}{m^{2} n \tau}\right), \\
& F_{1,1}^{(2)}(m, n ; \tau)=i^{n} \sum_{k=0}^{m n-1} q^{-\left(k+\frac{m n}{2}+\frac{1}{2}\right)^{2}} \sum_{\substack{r_{1}, \ldots, r_{n}=-\infty \\
2 m\left(r_{1}+\cdots+r_{n}\right)=m n+2 k}}^{\infty}(-1)^{r_{1}+\cdots+r_{n}} q^{m^{2} n\left(r_{1}^{2}+\cdots+r_{n}^{2}\right)}
\end{aligned}
$$

Proof Putting $y_{1}=y_{2}=\cdots=y_{n}$ in Corollary 3.3, we obtain Corollary 3.4.

\section{Corollary 3.5 For $n$ is even, we have}

$$
\sum_{k=0}^{n-1} q^{k^{2}} e^{2 k i z} \vartheta_{1}^{n}(z+k \pi \tau \mid n \tau)=F_{1,1}^{(1)}(n ; \tau) \vartheta_{3}(z \mid \tau)
$$




$$
\sum_{k=0}^{n-1} q^{k^{2}+k} e^{(2 k+1) i z} \prod_{j=1}^{n} \vartheta_{1}\left(z+\frac{\pi \tau}{2}+k \pi \tau \mid n \tau\right)=F_{1,1}^{(2)}(n ; \tau) \vartheta_{2}(z \mid \tau)
$$

where

$$
\begin{aligned}
& F_{1,1}^{(1)}(n ; \tau)=\frac{(\sqrt{-i})^{1-3 n} \sqrt{\tau^{1-n}}}{(\sqrt{n})^{n}} R_{1,1}^{(1)}\left(n ;-\frac{1}{n \tau}\right), \\
& F_{1,1}^{(1)}(n ; \tau)=\sum_{k=0}^{n-1}(-1)^{k} q^{-\left(k+\frac{n}{2}\right)^{2}} \sum_{\substack{r_{1}, \ldots, r_{n}=-\infty \\
2\left(r_{1}+\cdots+r_{n}\right)=n+2 k}}^{\infty} q^{n\left(r_{1}^{2}+\cdots+r_{n}^{2}\right)}, \\
& F_{1,1}^{(2)}(n ; \tau)=\frac{(\sqrt{-i})^{1-3 n} \sqrt{\tau^{1-n}}}{(\sqrt{n})^{n}} q^{-\frac{1}{4}} R_{1,1}^{(2)}\left(n ;-\frac{1}{n \tau}\right), \\
& F_{1,1}^{(2)}(n ; \tau)=\sum_{k=0}^{n-1}(-1)^{k} q^{-\left(k+\frac{n}{2}+\frac{1}{2}\right)^{2}} \sum_{\substack{r_{1}, \ldots, r_{n}=-\infty \\
2\left(r_{1}+\cdots+r_{n}\right)=n+2 k}}^{\infty} q^{n\left(r_{1}^{2}+\cdots+r_{n}^{2}\right)} .
\end{aligned}
$$

Proof Taking $m=1$ in Corollary 3.4, we obtain Corollary 3.5.

Corollary 3.6 Setting $m=1, n=2$ in (3.1), $y_{1}=y_{2}=p$, we have

$$
\begin{aligned}
& e^{2 p i z} \vartheta_{1}^{2}(z+p \pi \tau \mid 2 \tau)+q^{2 p+1} e^{2(p+1) i z} \vartheta_{1}^{2}(z+p \pi \tau+\pi \tau \mid 2 \tau) \\
& \quad=(-1)^{p}\left[\vartheta_{2}(2 p \pi \tau \mid 4 \tau)-q^{2 p+1} \vartheta_{2}((2 p+1) \pi \tau \mid 4 \tau) \vartheta_{3}(z \mid \tau)\right] .
\end{aligned}
$$

Corollary 3.7 Setting $m=1, n=2$ in (3.4), $y_{1}=y_{2}=\frac{2 p+1}{2}$, we have

$$
\begin{gathered}
e^{(2 p+1) i z} \vartheta_{1}^{2}\left(z+p \pi \tau+\frac{\pi \tau}{2} \mid 2 \tau\right)+q^{2 p+2} e^{(2 p+3) i z} \vartheta_{1}^{2}\left(z+p \pi \tau+\frac{3 \pi \tau}{2} \mid 2 \tau\right) \\
=(-1)^{p} q^{-\frac{1}{4}}\left[q^{-p} \vartheta_{2}(2 p \pi \tau \mid 4 \tau)-q^{p+1} \vartheta_{2}((2 p+1) \pi \tau \mid 4 \tau) \vartheta_{2}(z \mid \tau)\right] .
\end{gathered}
$$

Taking $p=0$ in (3.32) and (3.33), respectively, we deduce the following theta function identities:

$$
\begin{aligned}
& \vartheta_{1}^{2}(z \mid 2 \tau)+q e^{2 i z} \vartheta_{1}^{2}(z+\pi \tau \mid 2 \tau)=\vartheta_{2}(0 \mid 4 \tau)-q \vartheta_{2}(\pi \tau \mid 4 \tau) \vartheta_{3}(z \mid \tau) \\
& e^{i z} \vartheta_{1}^{2}\left(z+\frac{\pi \tau}{2} \mid 2 \tau\right)+q^{2} e^{3 i z} \vartheta_{1}^{2}\left(z+\frac{3 \pi \tau}{2} \mid 2 \tau\right) \\
& \quad=q^{-\frac{1}{4}}\left[\vartheta_{2}(0 \mid 4 \tau)-q \vartheta_{2}(\pi \tau \mid 4 \tau) \vartheta_{2}(z \mid \tau)\right] .
\end{aligned}
$$

Taking $p=1$ in (3.32) and (3.33), respectively, we deduce the following theta function identities:

$$
\begin{aligned}
& \vartheta_{4}^{2}(z \mid 2 \tau)-\vartheta_{1}^{2}(z \mid 2 \tau)=q \vartheta_{2}(2 \pi \tau \mid 4 \tau)-q^{4} \vartheta_{2}(3 \pi \tau \mid 4 \tau) \vartheta_{3}(z \mid \tau), \\
& \vartheta_{4}^{2}\left(z+\frac{\pi \tau}{2} \mid 2 \tau\right)-\vartheta_{1}^{2}\left(z+\frac{\pi \tau}{2} \mid 2 \tau\right) \\
& =q^{\frac{3}{4}} e^{-i z}\left[\vartheta_{2}(2 \pi \tau \mid 4 \tau)-q^{3} \vartheta_{2}(3 \pi \tau \mid 4 \tau) \vartheta_{2}(z \mid \tau)\right] .
\end{aligned}
$$


Taking $p=-1$ in (3.32) and (3.33), respectively, we deduce the following theta function identities:

$$
\begin{aligned}
& e^{-2 i z} \vartheta_{1}^{2}(z-\pi \tau \mid 2 \tau)+q^{-1} \vartheta_{1}^{2}(z \mid 2 \tau)=\vartheta_{2}(-2 \pi \tau \mid 4 \tau)+q^{-1} \vartheta_{2}(-\pi \tau \mid 4 \tau) \vartheta_{3}(z \mid \tau), \\
& e^{-i z} \vartheta_{1}^{2}\left(z-\frac{\pi \tau}{2} \mid 2 \tau\right)+e^{i z} \vartheta_{1}^{2}\left(z+\frac{\pi \tau}{2} \mid 2 \tau\right) \\
& \quad=-q^{-\frac{1}{4}}\left[q \vartheta_{2}(-2 \pi \tau \mid 4 \tau)-\vartheta_{2}(-\pi \tau \mid 4 \tau) \vartheta_{2}(z \mid \tau)\right] .
\end{aligned}
$$

Remark 3.8 Corollary 3.5 is an analogue of Ramanujan's circular summation formula Theorem 1.8 .

Remark 3.9 From Theorem 3.1 and Theorem 3.2 we may obtain more theta function identities.

\section{Further results and remarks}

In the present section, we give some special cases of Theorem 2.1 and derive some theta function identities.

Setting $p=0$ in Theorem 2.1, we have the following.

Corollary 4.1 Suppose that $n$ is even, $m$ is any positive integer; $y_{1}, y_{2}, \ldots, y_{n}$ are any complex numbers.

- When $y_{1}+y_{2}+\cdots+y_{n}=0$, we have

$$
\sum_{k=0}^{m n-1} \prod_{j=1}^{n} \vartheta_{1}\left(z+y_{j}+\frac{k \pi}{m n} \mid \tau\right)=R_{1,1}^{(1)}\left(y_{1}, y_{2}, \ldots, y_{n} ; m, n ; \tau\right) \vartheta_{3}\left(m n z \mid m^{2} n \tau\right)
$$

where

$$
R_{1,1}^{(1)}\left(y_{1}, y_{2}, \ldots, y_{n} ; m, n ; \tau\right)=m n q^{-\frac{n}{4}} \sum_{\substack{r_{1}, \ldots, r_{n}=-\infty \\ r_{1}+\cdots+r_{n}=\frac{n}{2}}}^{\infty} q^{r_{1}^{2}+\cdots+r_{n}^{2}} e^{-2 i\left(r_{1} y_{1}+\cdots+r_{n} y_{n}\right)}
$$

- When $y_{1}+y_{2}+\cdots+y_{n}=\frac{\pi}{2 m}$, we have

$$
\sum_{k=0}^{m n-1} \prod_{j=1}^{n} \vartheta_{1}\left(z+y_{j}+\frac{k \pi}{m n} \mid \tau\right)=R_{1,1}^{(2)}\left(y_{1}, y_{2}, \ldots, y_{n} ; m, n ; \tau\right) \vartheta_{4}\left(m n z \mid m^{2} n \tau\right)
$$

where

$$
R_{1,1}^{(2)}\left(y_{1}, y_{2}, \ldots, y_{n} ; m, n ; \tau\right)=m n q^{-\frac{n}{4}} e^{\frac{\pi i}{2 m}} \sum_{\substack{r_{1}, \ldots, r_{n}=-\infty \\ r_{1}+\cdots, r_{n}=\frac{n}{2}}}^{\infty} q^{r_{1}^{2}+\cdots+r_{n}^{2}} e^{-2 i\left(r_{1} y_{1}+\cdots+r_{n} y_{n}\right)}
$$

Corollary 4.2 Suppose that $n$ is even, $m$ is any positive integer, then

$$
\sum_{k=0}^{m n-1} \vartheta_{1}^{n}\left(z+\frac{k \pi}{m n} \mid \tau\right)=R_{1,1}^{(1)}(m, n ; \tau) \vartheta_{3}\left(m n z \mid m^{2} n \tau\right)
$$




$$
\sum_{k=0}^{m n-1} \vartheta_{1}^{n}\left(z+\frac{\pi}{2 m n}+\frac{k \pi}{m n} \mid \tau\right)=R_{1,1}^{(2)}(m, n ; \tau) \vartheta_{4}\left(m n z \mid m^{2} n \tau\right)
$$

where

$$
R_{1,1}^{(1)}(m, n ; \tau)=R_{1,1}^{(2)}(m, n ; \tau)=m n q^{-\frac{n}{4}} \sum_{\substack{r_{1}, \ldots, r_{n}=-\infty \\ r_{1}+\cdots+r_{n}=\frac{n}{2}}}^{\infty} q^{r_{1}^{2}+\cdots+r_{n}^{2}}
$$

Proof Taking $y_{1}=y_{2}=\cdots=y_{n}$ in Corollary 4.1, we get Corollary 4.2.

Corollary 4.3 For $m$ is any positive integer, we have

$$
\begin{aligned}
& \sum_{k=0}^{2 m-1} \vartheta_{1}^{2}\left(z+\frac{k \pi}{2 m} \mid \tau\right)=2 m \vartheta_{2}(0 \mid 2 \tau) \vartheta_{3}\left(2 m z \mid 2 m^{2} \tau\right) \\
& \sum_{k=0}^{2 m-1} \vartheta_{1}^{2}\left(z+\frac{\pi}{4 m}+\frac{k \pi}{2 m} \mid \tau\right)=2 m \vartheta_{2}(0 \mid 2 \tau) \vartheta_{4}\left(2 m z \mid 2 m^{2} \tau\right) .
\end{aligned}
$$

Proof Putting $n=2$ in Theorem 4.2 and noting that $R_{1,1}^{(1)}(m, n ; \tau)=R_{1,1}^{(2)}(m, n ; \tau)=2 m \vartheta_{2}(0$ $2 \tau)$, we get Corollary 4.3 .

Remark 4.4 Corollary 4.3 is an analogue of Boon's result [6, p. 3440, Eq. (10)].

Corollary 4.5 For $m$ is any positive integer, we have

$$
\begin{aligned}
& \sum_{k=0}^{4 m-1} \vartheta_{1}^{4}\left(z+\frac{k \pi}{4 m} \mid \tau\right)=R(m ; \tau) \vartheta_{3}\left(4 m z \mid 4 m^{2} \tau\right), \\
& \sum_{k=0}^{4 m-1} \vartheta_{1}^{4}\left(z+\frac{\pi}{8 m}+\frac{k \pi}{4 m} \mid \tau\right)=R(m ; \tau) \vartheta_{4}\left(4 m z \mid 4 m^{2} \tau\right),
\end{aligned}
$$

where

$$
R(m ; \tau)=4 m q^{-1} \sum_{\substack{r_{1}, \ldots, r_{4}=-\infty \\ r_{1}+\cdots+r_{4}=2}}^{\infty} q^{r_{1}^{2}+\cdots+r_{4}^{2}}
$$

Corollary 4.6 For $n$ is even, we have

$$
\begin{aligned}
& \sum_{k=0}^{n-1} \vartheta_{1}^{n}\left(z+\frac{k \pi}{n} \mid \tau\right)=R(\tau) \vartheta_{3}(n z \mid n \tau), \\
& \sum_{k=0}^{n-1} \vartheta_{1}^{n}\left(z+\frac{\pi}{2 n}+\frac{k \pi}{n} \mid \tau\right)=R(\tau) \vartheta_{4}(n z \mid n \tau),
\end{aligned}
$$

where

$$
R(\tau)=n q^{-\frac{n}{4}} \sum_{\substack{r_{1}, \ldots, r_{n}=-\infty \\ r_{1}+\cdots+r_{n}=\frac{n}{2}}}^{\infty} q^{r_{1}^{2}+\cdots+r_{n}^{2}}
$$


Proof Taking $m=1$ in Corollary 4.2, we get Corollary 4.6.

Setting $m=1$ in Theorem 2.1, we have the following.

Corollary 4.7 Suppose that $n$ is even, $p$ is any integer; $y_{1}, y_{2}, \ldots, y_{n}$ are any complex numbers.

- When $y_{1}+y_{2}+\cdots+y_{n}=p \pi$, we have

$$
\sum_{k=0}^{n-1} \prod_{j=1}^{n} \vartheta_{1}\left(z+y_{j}+\frac{k \pi}{n} \mid \tau\right)=R_{1,1}^{(1)}\left(y_{1}, y_{2}, \ldots, y_{n} ; n, p ; \tau\right) \vartheta_{3}(n z \mid n \tau)
$$

where

$$
R_{1,1}^{(1)}\left(y_{1}, y_{2}, \ldots, y_{n} ; n, p ; \tau\right)=(-1)^{p} n q^{-\frac{n}{4}} \sum_{\substack{r_{1}, \ldots, r_{n}=-\infty \\ r_{1}+\cdots+r_{n}=\frac{n}{2}}}^{\infty} q^{r_{1}^{2}+\cdots+r_{n}^{2}} e^{-2 i\left(r_{1} y_{1}+\cdots+r_{n} y_{n}\right)}
$$

- When $y_{1}+y_{2}+\cdots+y_{n}=\frac{(2 p+1) \pi}{2}$, we have

$$
\sum_{k=0}^{n-1} \prod_{j=1}^{n} \vartheta_{1}\left(z+y_{j}+\frac{k \pi}{n} \mid \tau\right)=R_{1,1}^{(2)}\left(y_{1}, y_{2}, \ldots, y_{n} ; n, p ; \tau\right) \vartheta_{4}(n z \mid n \tau)
$$

where

$$
R_{1,1}^{(2)}\left(y_{1}, y_{2}, \ldots, y_{n} ; n, p ; \tau\right)=(-1)^{p} n i q^{-\frac{n}{4}} \sum_{\substack{r_{1}, \ldots, r_{n}=-\infty \\ r_{1}+\cdots+r_{n}=\frac{n}{2}}}^{\infty} q^{r_{1}^{2}+\cdots+r_{n}^{2}} e^{-2 i\left(r_{1} y_{1}+\cdots+r_{n} y_{n}\right)}
$$

Setting $p=0$ in Corollary 4.7, we have the following.

Corollary 4.8 Suppose that $n$ is even; $y_{1}, y_{2}, \ldots, y_{n}$ are any complex numbers.

- When $y_{1}+y_{2}+\cdots+y_{n}=0$, we have

$$
\sum_{k=0}^{n-1} \prod_{j=1}^{n} \vartheta_{1}\left(z+y_{j}+\frac{k \pi}{n} \mid \tau\right)=R_{1,1}^{(1)}\left(y_{1}, y_{2}, \ldots, y_{n} ; n ; \tau\right) \vartheta_{3}(n z \mid n \tau)
$$

where

$$
R_{1,1}^{(1)}\left(y_{1}, y_{2}, \ldots, y_{n} ; n ; \tau\right)=n q^{-\frac{n}{4}} \sum_{\substack{r_{1}, \ldots, r_{n}=-\infty \\ r_{1}+\cdots+r_{n}=\frac{n}{2}}}^{\infty} q^{r_{1}^{2}+\cdots+r_{n}^{2}} e^{-2 i\left(r_{1} y_{1}+\cdots+r_{n} y_{n}\right)}
$$

- When $y_{1}+y_{2}+\cdots+y_{n}=\frac{\pi}{2}$, we have

$$
\sum_{k=0}^{n-1} \prod_{j=1}^{n} \vartheta_{1}\left(z+y_{j}+\frac{k \pi}{n} \mid \tau\right)=R_{1,1}^{(2)}\left(y_{1}, y_{2}, \ldots, y_{n} ; n ; \tau\right) \vartheta_{4}(n z \mid n \tau)
$$


where

$$
R_{1,1}^{(2)}\left(y_{1}, y_{2}, \ldots, y_{n} ; n ; \tau\right)=n i q^{-\frac{n}{4}} \sum_{\substack{r_{1}, \ldots, r_{n}=-\infty \\ r_{1}+\cdots+r_{n}=\frac{n}{2}}}^{\infty} q^{r_{1}^{2}+\cdots+r_{n}^{2}} e^{-2 i\left(r_{1} y_{1}+\cdots+r_{n} y_{n}\right)}
$$

Corollary 4.9 Suppose that $n$ is even, $m$ is any positive integer, $a, b$ are any nonnegative integers and $a+b=n, p$ is any integer; $x, y$ are any complex numbers.

- When $a x+b y=\frac{p \pi}{m}$, we have

$$
\begin{aligned}
& \sum_{k=0}^{m n-1} \vartheta_{1}^{a}\left(z+x+\frac{k \pi}{m n} \mid \tau\right) \vartheta_{1}^{b}\left(z+y+\frac{k \pi}{m n} \mid \tau\right) \\
& =R_{1,1}^{(1)}(x, y ; m, n, p ; \tau) \vartheta_{3}\left(m n z \mid m^{2} n \tau\right),
\end{aligned}
$$

where

$$
\begin{aligned}
& R_{1,1}^{(1)}(x, y ; m, n, p ; \tau) \\
& =m n q^{-\frac{n}{4}} e^{\frac{p \pi i}{m}-n y i} \sum_{\substack{r_{1}, \ldots, r_{a}, s_{1}, \ldots, s_{b}=-\infty \\
r_{1}+\cdots+r_{a}+s_{1}+\cdots+s_{b}=\frac{n}{2}}}^{\infty} q^{r_{1}^{2}+\cdots+r_{a}^{2}+s_{1}^{2}+\cdots+s_{b}^{2}} e^{-2 i\left(r_{1}+\cdots+r_{a}\right)(x-y)} .
\end{aligned}
$$

- When $a x+b y=\frac{(2 p+1) \pi}{2 m}$, we have

$$
\begin{aligned}
& \sum_{k=0}^{m n-1} \vartheta_{1}^{a}\left(z+x+\frac{k \pi}{m n} \mid \tau\right) \vartheta_{1}^{b}\left(z+y+\frac{k \pi}{m n} \mid \tau\right) \\
& =R_{1,1}^{(2)}(x, y ; m, n, p ; \tau) \vartheta_{4}\left(m n z \mid m^{2} n \tau\right),
\end{aligned}
$$

where

$$
\begin{aligned}
& R_{1,1}^{(2)}(x, y ; m, n, p ; \tau) \\
& =m n q^{-\frac{n}{4}} e^{\frac{(2 p+1) \pi i}{2 m}-n y i} \sum_{\substack{r_{1}, \ldots, r_{a}, s_{1}, \ldots, s_{b}=-\infty \\
r_{1}+\cdots+r_{a}+s_{1}+\cdots+s_{b}=\frac{n}{2}}}^{\infty} q^{r_{1}^{2}+\cdots+r_{a}^{2}+s_{1}^{2}+\cdots+s_{b}^{2}} e^{-2 i\left(r_{1}+\cdots+r_{a}\right)(x-y)} .
\end{aligned}
$$

Proof Setting $y_{1}=y_{2}=\cdots=y_{a}=x$ and $y_{a+1}=y_{a+2}=\cdots=y_{n}=y$ with $a+b=n$ in Theorem 2.1, we obtain Corollary 4.9

Corollary 4.10 Suppose that $m$ is any positive integer.

$$
\begin{aligned}
& \sum_{k=0}^{2 m-1} \vartheta_{1}\left(z+x+\frac{k \pi}{2 m} \mid \tau\right) \vartheta_{1}\left(z-x+\frac{k \pi}{2 m} \mid \tau\right)=2 m \vartheta_{2}(2 x \mid 2 \tau) \vartheta_{3}\left(2 m z \mid 2 m^{2} \tau\right) \\
& \sum_{k=0}^{2 m-1} \vartheta_{1}\left(z+x+\frac{k \pi}{2 m} \mid \tau\right) \vartheta_{1}\left(z-x+\frac{(k+1) \pi}{2 m} \mid \tau\right) \\
& =2 m \vartheta_{2}\left(2 x-\frac{\pi}{2 m} \mid 2 \tau\right) \vartheta_{4}\left(2 m z \mid 2 m^{2} \tau\right)
\end{aligned}
$$


Proof Taking $p=0, a=b=1$ in Corollary 4.9, we have

$$
\begin{aligned}
& R_{1,1}^{(1)}(x ; m ; \tau)=2 m q^{-\frac{1}{2}} e^{2 x i} \sum_{\substack{r_{1}, s_{1}=-\infty \\
r_{1}+s_{1}=1}}^{\infty} q^{r_{1}^{2}+s_{1}^{2}} e^{-4 i r_{1} x}=2 m \vartheta_{2}(2 x \mid 2 \tau), \\
& R_{1,1}^{(2)}(x, y ; m ; \tau)=2 m q^{-\frac{1}{2}} e^{2 i x-\frac{\pi i}{2 m}} \sum_{\substack{r_{1}, s_{1}=-\infty \\
r_{1}+s_{1}=1}}^{\infty} q^{r_{1}^{2}+s_{1}^{2}} e^{-2 i r_{1}\left(2 x-\frac{\pi}{2 m}\right)}=2 m \vartheta_{2}\left(2 x-\frac{\pi}{2 m} \mid 2 \tau\right)
\end{aligned}
$$

and noting that $x+y=0$ and $x+y=\frac{\pi}{2 m}$, we obtain Corollary 4.10.

Taking $m=1$ in Corollary 4.10, we have

$$
\begin{aligned}
& \vartheta_{1}(z+x \mid \tau) \vartheta_{1}(z-x \mid \tau)+\vartheta_{2}(z+x \mid \tau) \vartheta_{2}(z-x \mid \tau)=2 \vartheta_{2}(2 x \mid 2 \tau) \vartheta_{3}(2 z \mid 2 \tau) \\
& \vartheta_{1}(z+x \mid \tau) \vartheta_{2}(z-x \mid \tau)-\vartheta_{2}(z+x \mid \tau) \vartheta_{1}(z-x \mid \tau)=2 \vartheta_{1}(2 x \mid 2 \tau) \vartheta_{4}(2 z \mid 2 \tau)
\end{aligned}
$$

Taking $m=2$ in Corollary 4.10, we have

$$
\begin{aligned}
& \vartheta_{1}(z+x \mid \tau) \vartheta_{1}(z-x \mid \tau)+\vartheta_{2}(z+x \mid \tau) \vartheta_{2}(z-x \mid \tau) \\
& \quad+\vartheta_{1}\left(z+x+\frac{\pi}{4} \mid \tau\right) \vartheta_{1}\left(z-x+\frac{\pi}{4} \mid \tau\right)+\vartheta_{1}\left(z+x-\frac{\pi}{4} \mid \tau\right) \vartheta_{1}\left(z-x-\frac{\pi}{4} \mid \tau\right) \\
& =4 \vartheta_{2}(2 x \mid 2 \tau) \vartheta_{3}(4 z \mid 8 \tau), \\
& \vartheta_{1}(z+x \mid \tau) \vartheta_{1}\left(z-x+\frac{\pi}{4} \mid \tau\right)+\vartheta_{1}\left(z+x+\frac{\pi}{4} \mid \tau\right) \vartheta_{2}(z-x \mid \tau) \\
& \quad-\vartheta_{2}(z+x \mid \tau) \vartheta_{1}\left(z-x-\frac{\pi}{4} \mid \tau\right)+\vartheta_{1}\left(z+x-\frac{\pi}{4} \mid \tau\right) \vartheta_{1}(z-x \mid \tau) \\
& =4 \vartheta_{2}\left(2 x-\frac{\pi}{4} \mid 2 \tau\right) \vartheta_{4}(4 z \mid 8 \tau) .
\end{aligned}
$$

Remark 4.11 We can obtain the corresponding results of $\vartheta_{2}(z \mid \tau)$ from Theorem 2.2, we here omit them.

Remark 4.12 No doubt more theta function identities may be formulated from Theorem 2.2 and other theorems and corollaries.

\section{Conclusion}

Ramanujan's circular summation is an interesting subject in his notebook. In this paper, we obtain two Ramanujan-type circular summation formulas. We also give the corresponding imaginary transformation formulas for Ramanujan-type circular summations and some identities of the classical Jacobi theta functions $\vartheta_{1}(z \mid \tau)$ and $\vartheta_{2}(z \mid \tau)$. 
Availability of data and materials

Not applicable.

\section{Competing interests}

The authors declare that they have no competing interests.

\section{Authors' contributions}

There was an equal amount of contributions from all authors. All authors read and approved the final manuscript.

\section{Author details}

'School of Intelligent Technology and Engineering, Chongqing University of Science and Technology, Chongqing Higher Education Mega Center, Huxi Campus, Chongqing 401331, People's Republic of China. ${ }^{2}$ Department of Mathematics, Chongqing Normal University, Chongqing Higher Education Mega Center, Huxi Campus, Chongqing 401331, People's Republic of China.

\section{Publisher's Note}

Springer Nature remains neutral with regard to jurisdictional claims in published maps and institutional affiliations.

Received: 28 July 2020 Accepted: 11 November 2020 Published online: 09 December 2020

\section{References}

1. Ahlgren, S.: The sixth, eighth, ninth and tenth powers of Ramanujan theta function. Proc. Am. Math. Soc. 128, 1333-1338 (2000)

2. Andrews, G.E., Berndt, B.C.: Ramanujan's Lost Notebook Part III. Springer, New York (2012)

3. Bellman, R.: A Brief Introduction to Theta Functions. Holt, Rinehart \& Winston, New York (1961)

4. Berndt, B.C.: Ramanujan's Notebooks Part III. Springer, New York (1991)

5. Berndt, B.C.: Ramanujan's Notebooks Part V. Springer, New York (1998)

6. Boon, M., Glasser, M.L., Zak, J., Zucker, I.J.: Additive decompositions of $\theta$-functions of multiple arguments. J. Phys. A, Math. Gen. 15, 3439-3440 (1982)

7. Borwein, J.M., Borwein, P.B.: A cubic counterpart of Jacobi's identity and the AGM. Trans. Am. Math. Soc. 323, 691-701 (1991)

8. Cai, Y., Bi, Y.-Q., Luo, Q.-M.: Some new circular summation formulas of theta functions. Integral Transforms Spec. Funct. 25, 497-507 (2014)

9. Cai, Y., Chen, S., Luo, Q.-M.: Some circular summation formulas for the theta functions. Bound. Value Probl. 2013, 59 (2013)

10. Chan, H.H., Liu, Z.-G., Ng, S.T.: Circular summation of theta functions in Ramanujan's Lost Notebook. J. Math. Anal. Appl. 316, 628-641 (2006)

11. Chan, S.H., Liu, Z.-G.: On a new circular summation of theta functions. J. Number Theory 130, 1190-1196 (2010)

12. Chandrasekharan, K.: Elliptic Functions. Springer, Berlin (1985)

13. Chua, K.S.: Circular summation of the 13th powers of Ramanujan's theta function. Ramanujan J. 5, 353-354 (2001)

14. Chua, K.S.: The root lattice $A_{n}^{*}$ and Ramanujan's circular summation of theta functions. Proc. Am. Math. Soc. 130, 1-8 (2002)

15. Liu, X.-F., Luo, Q.-M.: A note for alternating Ramanujan's circular summation formula. Forum Math. 27, 3189-3203 (2015)

16. Liu, Z.-G.: Some inverse relations and theta function identities. Int. J. Number Theory 8, 1977-2002 (2012)

17. Luo, Q.-M.: A note for Ramanujan's circular summation formula. Integral Transforms Spec. Funct. 26, 567-585 (2015)

18. Montaldi, E., Zucchelli, G.: Additive decomposition for the product of two $\vartheta_{3}$ functions and modular equations. J. Math. Phys. 30, 2012-2015 (1989)

19. Murayama, T:: On an identity of theta functions obtained from weight enumerators of linear codes. Proc. Jpn. Acad., Ser. A, Math. Sci. 74, 98-100 (1998)

20. Ono, K.: On the circular summation of the eleventh powers of Ramanujan's theta function. J. Number Theory 76 , 62-65 (1999)

21. Ramanujan, S.: The Lost Notebook and Other Unpublished Papers. Narosa, New Delhi (1988)

22. Rangachari, S.S.: On a result of Ramanujan on theta functions. J. Number Theory 48, 364-372 (1994)

23. Shen, L.C.: On the additive formula of the theta functions and a collection of Lambert series pertaining to the modular equations of degree 5. Trans. Am. Math. Soc. 345, 323-345 (1994)

24. Son, S.H.: Circular summation of theta functions in Ramanujan's Lost Notebook. Ramanujan J. 8, 235-272 (2004)

25. Whittaker, E.T., Watson, G.N.: A Course of Modern Analysis, 4th edn. Cambridge University Press, Cambridge (1927) (Reprinted 1963)

26. Xu, P.: An elementary proof of Ramanujan's circular summation formula and its generalizations. Ramanujan J. 27, 409-417 (2012)

27. Zeng, X.-F.: A generalized circular summation of theta function and its application. J. Math. Anal. Appl. 356, 698-703 (2009)

28. Zhou, Y., Luo, Q.-M.: Circular summation formulas for theta function $\vartheta_{4}(z \mid \tau)$. Adv. Differ. Equ. 2014, 243 (2014)

29. Zhu, J.-M.: An alternate circular summation formula of theta functions and its applications. Appl. Anal. Discrete Math. 6, 114-125 (2012)

30. Zhu, J.-M.: A note on a generalized circular summation formula of theta functions. J. Number Theory 132, 1164-1169 (2012) 